\title{
A rare cause of a common symptom, Anakinra is effective in the urticaria of Schnitzler Syndrome: a case report Lisa A Devlin*1, Gary Wright ${ }^{2}$ and J David M Edgar ${ }^{1}$
}

\author{
Address: ${ }^{1}$ Regional Immunology Service, Royal Hospitals, The Belfast Trust, Grosvenor Road, Belfast BT12 6BN and ${ }^{2}$ Department of \\ Rheumatology, Royal Hospitals, The Belfast Trust, Grosvenor Road, Belfast BT12 6BN \\ Email: Lisa A Devlin* - lisa.devlin1@belfasttrust.hscni.net; Gary Wright - gary.wright@belfasttrust.hscni.net; J \\ David M Edgar - david.edgar@belfasttrust.hscni.net \\ * Corresponding author
}

Published: 24 November 2008

Cases Journal 2008, I:348 doi:10.1186/1757-1626-1-348

This article is available from: http://www.casesjournal.com/content/l/I/348

(C) 2008 Devlin et al; licensee BioMed Central Ltd.

This is an Open Access article distributed under the terms of the Creative Commons Attribution License (http://creativecommons.org/licenses/by/2.0), which permits unrestricted use, distribution, and reproduction in any medium, provided the original work is properly cited.
Received: II November 2008

Accepted: 24 November 2008

\begin{abstract}
Introduction: Schnitzler Syndrome is an uncommon, inflammatory condition that presents with a constellation of chronic unremitting urticaria, fever, bone pain, arthralgia or arthritis, and a monoclonal IgM gammopathy. There is usually neutrophilia and raised inflammatory markers. Delayed diagnosis is common and treatment often unsuccessful.

Case presentation: We report the case of a 43-year-old caucasian man who presented with urticaria unresponsive to conventional therapy. There was considerable delay in recognition of this as Schnitzler Syndrome, and symptoms were unresponsive to conventional immunosuppressive therapy.

Commencement of anakinra was associated with a rapid and sustained clinical response.

Conclusion: Schnitzler Syndrome is a rare disorder that mimics chronic idiopathic urticaria. This diagnosis should be considered in patients with urticaria unresponsive to antihistamines and conventional immunosuppressive therapy. Anakinra is an effective treatment although further studies are required, to determine long term therapeutic requirements and assess any potential adverse effects.
\end{abstract}

\section{Introduction}

Chronic idiopathic urticaria (CIU) is a common, benign condition for which patients are frequently referred to allergy or dermatology clinics. The investigation and management of patients with urticaria has recently been reviewed [1]. The majority of patients presenting with urticaria do not require extensive laboratory investigation and their symptoms usually respond to regular oral antihistamines, albeit often at high dose. For selected unresponsive patients, the use of immunosuppressive agents is occasionally required. The case we report initially pre- sented with an urticarial rash only, but this was later followed by the development of fever, arthralgia, lymphadenopathy and raised inflammatory markers. Extensive investigation was therefore undertaken and a provisional diagnosis of Adult Onset Still's Disease (AOSD) made. However in view of the lack of response to immunosuppressive treatment and the subsequent dramatic response to anakinra, the final diagnosis of Schnitzler Syndrome (SS) was established. SS is a rare condition, with less than 100 cases described. Because of its rarity and the lack of a specific diagnostic test, it is likely to be 
under diagnosed. This case highlights its importance as a differential diagnosis of CIU and its apparent exquisite sensitivity to treatment with anakinra.

\section{Case presentation}

A previously well 43-year-old man presented with chronic urticaria (Figure 1). There was no obvious precipitant identified from the history, he was systemically well and investigations including FBP, U\&E, LFTs, TFTs, ESR, and CRP were normal. A polyclonal increase in IgG and IgA was noted with an associated small $(<1 \mathrm{~g} / \mathrm{L})$ IgM kappa paraprotein. The rash was resistant to conventional treatment with non-sedating antihistamines.

At review, 6 months later, he had developed generalized fatigue, fever, night sweats, arthralgia, right knee and tibial pain, weight loss and bilateral axillary and inguinal lymphadenopathy.

Investigations showed a persistent neutrophilia $(>15 \times$ $10^{9} / \mathrm{L}$ ) and raised ESR and CRP. Other haematological indices were within normal limits. A persistent polyclonal elevation in IgG, A and $M$ was noted, with the IgM kappa paraprotein increased to $3.3 \mathrm{~g} / \mathrm{L}$. Serum ferritin was mildly elevated (361 $\mu \mathrm{g} / \mathrm{L}$, normal range 18-325). An inflammatory arthritis was suspected, and admission arranged for further investigation and treatment.

Quotidian spiking fevers $\left(>38^{\circ} \mathrm{C}\right)$ were recorded whilst an inpatient. Isotope bone scan showed increased tracer uptake at proximal aspect of right tibia in comparison with the left. MRI right knee showed non specific marrow signal change at distal end of femur/proximal tibia. CT chest, abdomen and pelvis showed florid bilateral axillary and inguinal lymphadenopathy (Figure 2). Serum antibodies to nuclei, extractable nuclear antigens, double stranded DNA, cyclic citrullinated peptide, and neutrophil cytoplasmic antigens were all negative. Rheumatoid factor was negative and serum C3 and C4 levels were normal. There was a slight elevation in $\mathrm{B} 2$ microglobulin and plasma viscosity, but lymphocyte subsets including kappa/lambda ratios were normal, and urine analysis was negative for Bence Jones protein. Serological investigations for EBV, CMV, HIV, syphilis, hepatitis A, B and C,

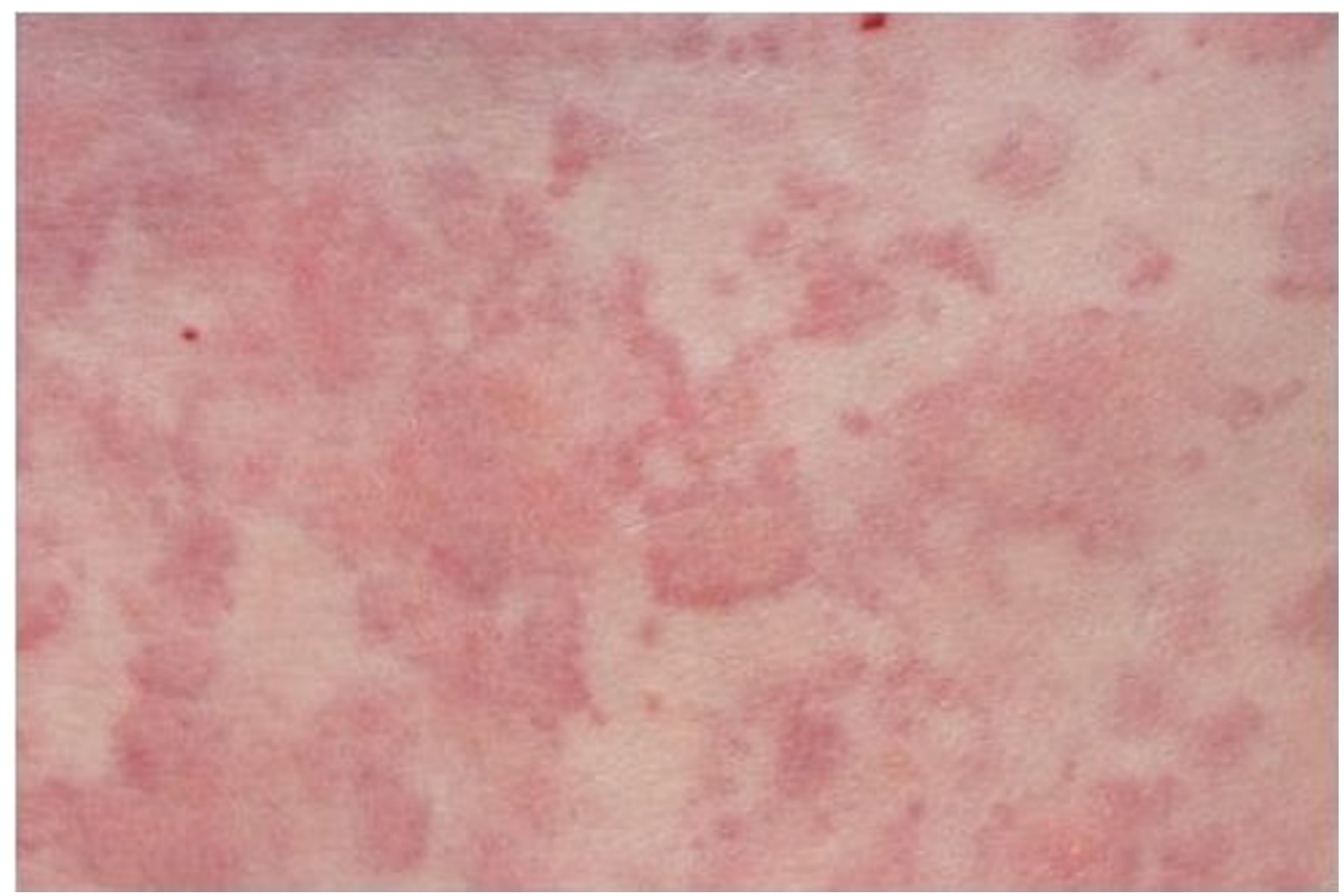

Figure I

Urticarial rash at presentation. 


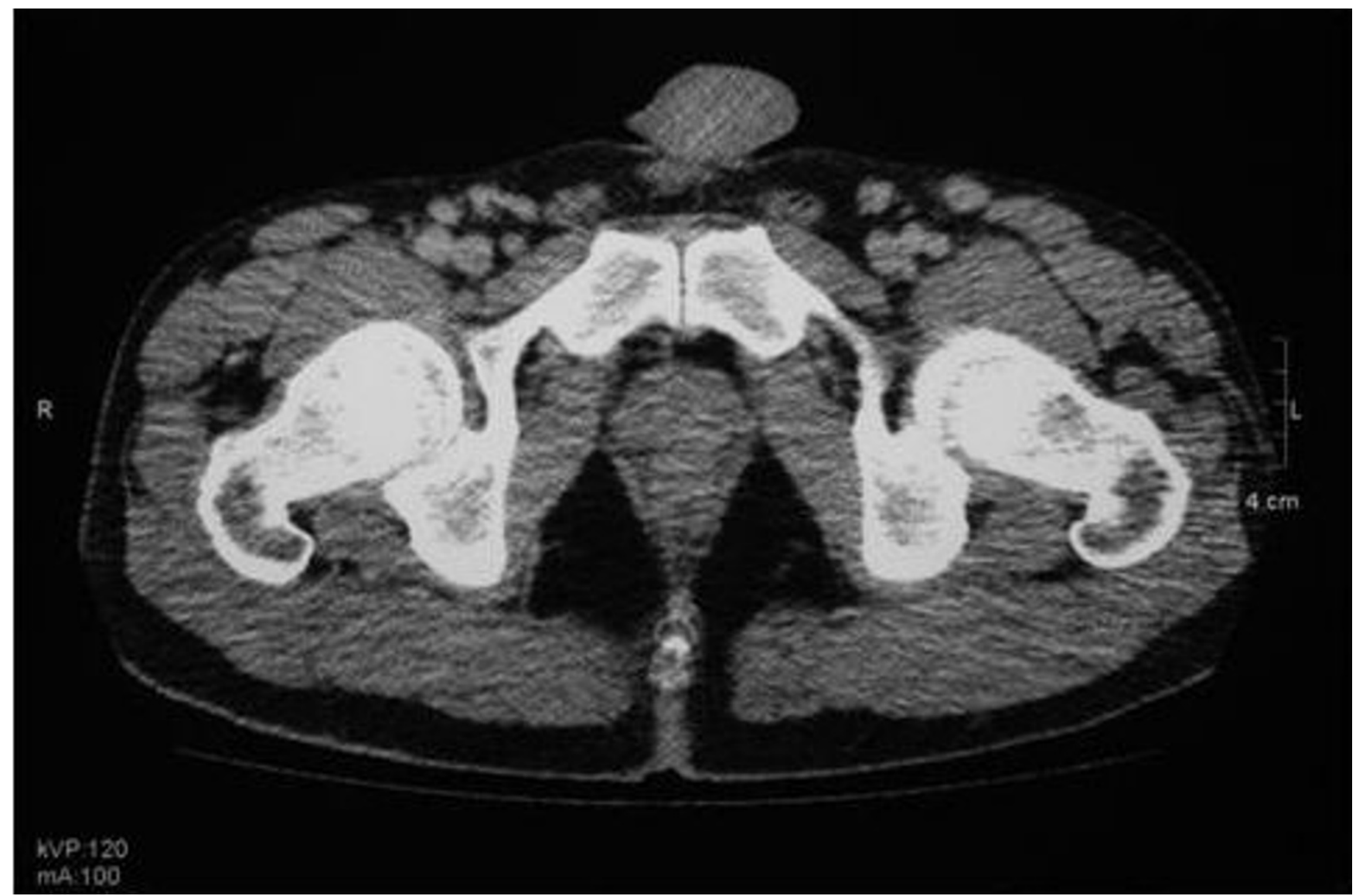

Figure 2

CT scan of pelvis indicating multiple enlarged lymph nodes.

borrelia burgdoderi, brucella abortus, chlamydia, Q fever, mycoplasma, and HHV8 were negative. ASOT was $<200$. Urine cultures for mycobacterium were repeatedly negative.

Skin biopsy demonstrated an inflammatory infiltrate in the epidermis, suggestive of either rheumatoid arthritis or Still's disease. Lymph node biopsy revealed polyclonal reactive hyperplasia with abundance of plasma cells (Figure 3). Castleman disease was considered but excluded after a further biopsy and review by several expert histopathologists. Bone marrow biopsy showed reactive changes only.

Mutations were not found in the coding regions of MVK, TNFRSF1A, and NLRP3 (previously NALP3/CIAS1) making the diagnoses of Hyper IgD syndrome, TNF receptor associated periodic syndrome (TRAPS), and Muckle Wells/Familial Cold Urticaria all unlikely.

A tentative diagnosis of Adult Onset Still's Disease (AOSD) was made. Trials of etoricoxib, and subsequent oral prednisolone (40 mg daily) with methotrexate (20 mg weekly) were however ineffective. Two cycles of pulsed methylprednisolone $(250 \mathrm{mg}$ and $500 \mathrm{mg}$ ) resulted in transient improvement in both symptoms and inflammatory markers over the initial 36 hours, but symptoms returned on completion of cycle. A trial of infliximab $(5 \mathrm{mg} / \mathrm{kg})$ resulted in a paradoxical exacerbation with night sweats, fever, arthralgia and urticaria.

Symptoms and signs persisted over the next year despite symptomatic treatment. The patient was severely disabled by these and only able to work part time.

Four years after initial presentation, a further trial of prednisolone $(60 \mathrm{mg} /$ day) was commenced. 48 hours after commencement of treatment there was almost complete resolution of symptoms. Initial CRP $(149 \mathrm{mg} / \mathrm{L})$ fell to $10.8 \mathrm{mg} / \mathrm{L}$ after 13 days treatment. Azathioprine was commenced as a steroid sparing agent however the development of severe arthralgia, joint stiffness, rash, and fever with a CRP of $164 \mathrm{mg} / \mathrm{L}$ led to its withdrawal after 9 days. Mycophenolate mofetil (1 g BD) was substituted however 


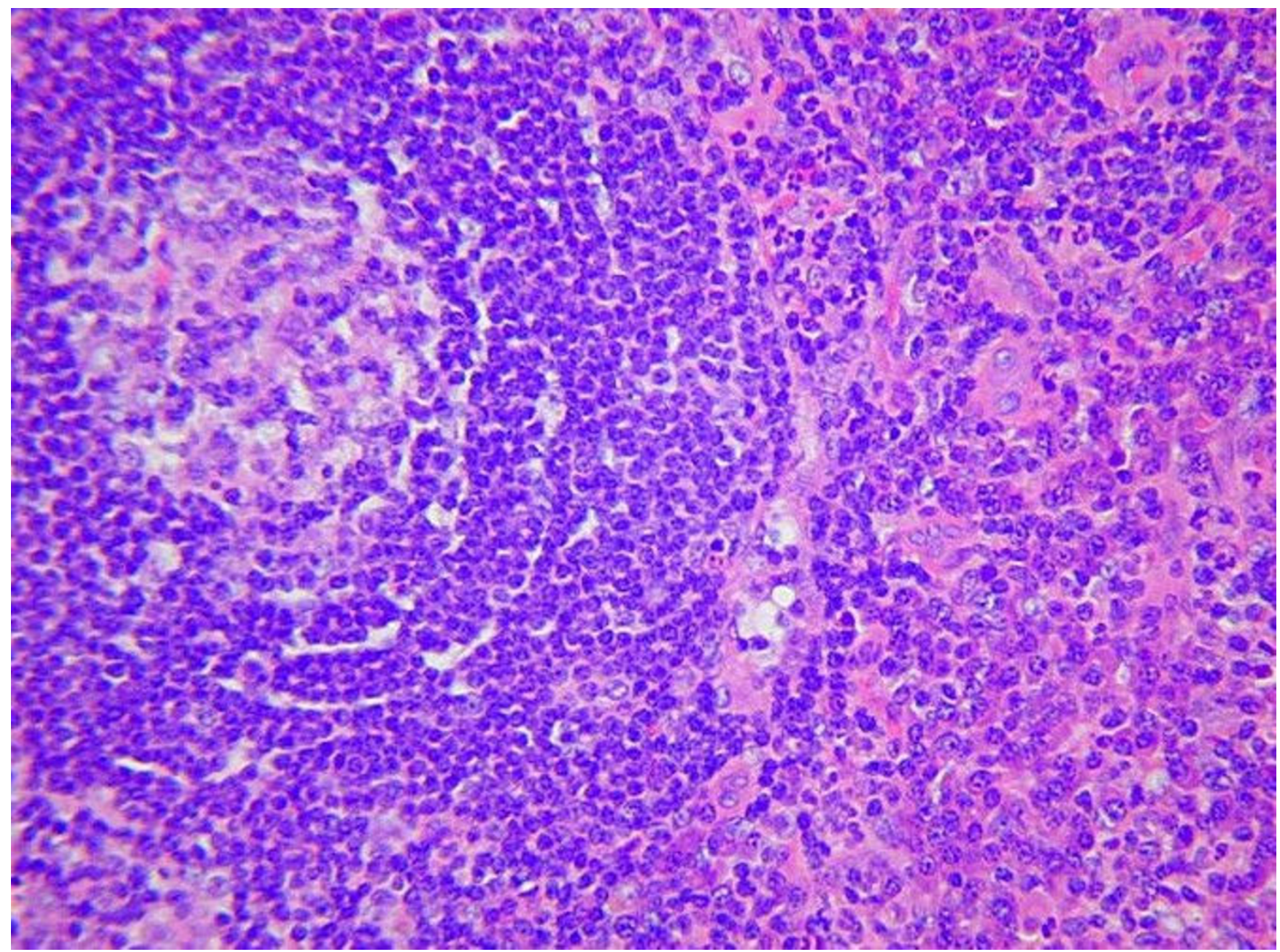

Figure 3

Lymph node biopsy demonstrates widespread infiltration by plasma cells.

despite 3 months treatment; prednisolone dose could not be reduced below $50 \mathrm{mg} /$ day.

Given the unresponsiveness of this patient's disease to a number of immunosuppressant drugs, and reports of a beneficial response in refractory AOSD [2], a trial of anakinra, (100 mg/day) was commenced. Within 24 hours there was almost complete resolution of symptoms. CRP normalized within 7 days. The patient has remained on anakinra therapy for 16 months with continued symptom control. Temporary interruption of treatment on two occasions was associated with recurrence of rash, fever and arthralgia within 24 hours. Symptoms quickly resolved on recommencing anakinra. In light of the clini-

Table I: Diagnostic Criteria for Schnitzler Syndrome*

\author{
Urticarial skin rash, monoclonal lgM component, and at least 2 of the following criteria: \\ Fever \\ Arthralgia or arthritis \\ Bone pain \\ Lymphadenopathy \\ Hepato- and/or splenomegaly \\ Elevated ESR \\ Leucocytosis \\ Abnormal findings on bone morphological investigations \\ *Another cause must be eliminated in all cases, most notably hyper IgD syndrome, AOSD, hypocomplementaemic urticarial vasculitis, acquired CI \\ inhibitor deficiency, and cryoglobulinaemia.
}


cal course and following an extensive literature review, the final diagnosis of SS was made. The patient fulfilled both essential criteria and 6 of the additional criteria established by Lipsker et al (Table 1) [3].

\section{Discussion}

SS is an uncommon syndrome with only 94 cases reported in the literature [4]. It was first described by L. Schnitzler [5], as a clinical entity consisting of chronic urticaria, fever, bone pain, arthralgia or arthritis, and a monoclonal IgM gammopathy.

This patient was at first thought to have CIU but failed to respond to conventional therapy. The subsequent development of systemic symptoms led to a wide differential being considered and several specialists reviewed his case. Because of the prominence of joint symptoms, AOSD was the provisional diagnosis for some time. It is important to note that the patient met the diagnostic criteria for AOSD established by Yamaguchi et al and that these are not therefore entirely specific and potential remains for diagnostic confusion between the two conditions [6]. Specific diagnostic tests do not exist for either AOSD or SS, however serum ferritin levels detected in AOSD are usually higher than in this patient and the presence of a paraprotein (typically IgM kappa) is characteristic of SS. Skin, lymph node and bone marrow biopsies in SS often show non specific 'reactive changes' on histopathology.

SS is a rare chronic condition and there are no reports of spontaneous remission [3]. The mean age of onset is 51 years, diagnostic delay is common and greater than 5 years in most cases [3]. In the recent review of 94 published cases, the prognosis was regarded as favorable with $91 \%$ survival after 15 years [4]. A significant long term complication (15\%) remains the development of lymphoproliferative disease, especially Waldenstroms macroglobulinaemia $10-20$ years following the onset of symptoms $[3,4] .3$ patients developed amyloidosis in that series [4].

Chronic urticaria, unresponsive to antihistamines is characteristic of SS and is an important clinical message of this case. Oral steroids have not been beneficial at acceptable doses, and given the chronicity of the disease, are associated with significant cumulative side effects [4]. Despite the significant evidence of inflammation in this condition, there is almost complete failure to respond to a variety of immunosuppressants including azathioprine, ciclosporin, methotrexate, high dose immunoglobulin, hydroxychloroquine, dapsone, colchicine, rituximab, mycophenolate and infliximab [7-10]. Until recently, therefore, treatment of SS has been associated with a very disappointing outcome.
Recently, anakinra, an IL-1 receptor antagonist has been associated with an excellent outcome [7-10]. Within 24 hours of commencing anakinra, our patient had almost complete resolution of his symptoms, and has since been able to return to work full time. Discontinuation of anakinra for 1 day, on two occasions, was associated with a rapid disabling return of symptoms.

Six patients with SS have to date made a complete response, both symptomatically, and with a reduction in inflammatory markers and white cell count, to anakinra [7-10]. The longest published follow up is 18 months, our patient has been treated for 21 months and continues to require therapy. No patient has to our knowledge discontinued anakinra and remained in remission. It therefore appears likely that long term treatment will be required, however periodic withdrawal of therapy seems reasonable to assess current disease activity.

Anakinra has been well tolerated, with no significant side effects in either our patient or other cases of SS reported to date. One patient developed a mild reaction at the injection site which resolved after few weeks [8], and one patient had an injection associated headache [10], which subsided with ongoing therapy.

The pathogenesis of SS is essentially unknown. The reported universal response to anakinra suggests a key role of IL-1 in the pathophysiology.

Interestingly, anakinra has also been reported to provide beneficial effects in patients with Muckle Wells syndrome [11]. Given that there is also considerable overlap in the clinical features of SS and Muckle Wells syndrome, this raises the possibility of common pathophysiology. One published case of SS had molecular analysis carried out for mutations in the coding region of NALP3 (the defect associated with Muckle Wells syndrome), but no mutations were identified [7]. With increasing recognition and characterization of patients with SS, the possibility that it forms part of the spectrum of cryopyrin diseases remains.

\section{Conclusion}

SS is a rare cause of a common symptom (urticaria), but this typically occurs in patients with other systemic symptoms. These systemic symptoms show considerable overlap with AOSD and therefore immunologists, dermatologists and rheumatologists all need to be aware of this potential diagnosis. The presence of an IgM paraprotein is an important indicator in favour of a diagnosis of SS.

Patients with SS have a significant risk of late malignancy and should be carefully monitored for its development. Until now, treatment has largely been unsuccessful, how- 
ever the introduction of anakinra has been very effective in this and 4 other published cases. Our experience suggests that treatment with anakinra is likely to be required in the long term in order to control symptoms, reduce inflammation and diminish the risk of long term side effects.

\section{Competing interests}

The authors declare that they have no competing interests.

\section{Authors' contributions}

LAD collated clinical data and drafted the initial manuscript. JDME provided clinical data, revised and amended the manuscript. GW provided clinical data and reviewed the manuscript. All authors have read and approved the final manuscript.

\section{Consent}

Written informed consent was obtained from the patient for publication of this case report and accompanying images. A copy of the written consent is available for review by the Editor-in-Chief of this journal

\section{References}

I. Powell RJ, Du Toit GL, Siddique N, Leech SC, Dixon TA, Clark AT, Mirakian R, Walker SM, Huber PA, Nasser SM: British Society for Allergy and Clinical Immunology (BSACI). BSACI guidelines for the management of chronic urticaria and angio-oedema. Clin Exp Allergy 2007, 37:63I-650.

2. Haraoui B, Bourrelle D, Kaminska E: Ankinra in the treatment of adult onset Still's disease. Ann Rheum Dis 2004, 63(suppl):28I.

3. Lipsker D, Veran Y, Grunenberger F, Cribier B, Heid E, Grosshans E: The Schnitzler Syndrome. Four Cases and Review of the Literature. Medicine 200I, 80:37-44.

4. de Koning HD, Bodar EJ, Meer JWM van der, Simon A, Schnitzler Syndrome Study Group: Schnitzler Syndrome: Beyond the Case Reports: Review and Follow-Up of 94 patients with an Emphasis on Prognosis and Treatment. Semin Arthritis Rheum 2007, 37:137-48.

5. Schnitzler L, Schubert B, Boasson M, Gardais J, Tourmen A: Urticaire chronique, lesions osseuses, macroglobulinemie IgM: maladie de Waldenstrom? 2eme presentation. Bull Soc Fr Dermatol Syphiligr 1974, 81:363.

6. Yamaguchi M, Ohta A, Tsunematsu T, Kasukawa R, Mizushima $Y$, Kashiwagi H, Kashiwazaki S, Tanimoto K, Matsumoto Y, Ota T, et al.: Preliminary criteria for classification of adult Still's disease. J Rheumatol 1992, 19(3):424-430.

7. Martinez-Taboada VM, Fontalba A, Blanco R, Fernandez-Luna JL: Successful treatment of refractory Schnitzler syndrome with anakinra: comment on the article by Hawkins et al. Arthritis Rheum 2005, 52:2226-8.

8. de Koning HD, Bodar EJ, Simon A, Hilst JHC van der, Netea MG, Meer JWM van der: Beneficial response to anakinra and thalidomide in Schnitzler's syndrome. Arch Rheum Dis 2006, 65:542-4.

9. Eiling E, Moller M, Kreiselmaier I, Brasch J, Schwarz T: Schnitzler syndrome: Treatment failure to rituximab but response to anakinra. J Am Acad Dermatol in press.

10. Schneider SW, Gaubitz M, Luger TA, Bonsmann G: Prompt response of refractory Schnitzler syndrome to treatment with anakinra. I Am Acad Dermatol 2007, 56:SI20-SI22.

II. Hawkins PN, Lachmann HJ, Aganna E, Mc Dermott MF: Spectrum of clinical features in Muckle-Wells Syndrome and Response to Anakinra. Arthritis Rheum 2004, 50:607-I2.
Publish with Biomed Central and every scientist can read your work free of charge

"BioMed Central will be the most significant development for disseminating the results of biomedical research in our lifetime. "

Sir Paul Nurse, Cancer Research UK

Your research papers will be:

- available free of charge to the entire biomedical community

- peer reviewed and published immediately upon acceptance

- cited in PubMed and archived on PubMed Central

- yours - you keep the copyright

Submit your manuscript here:

http://www.biomedcentral.com/info/publishing_adv.asp
BioMedcentral 